\title{
Multifocal Intraocular Lens Differentiation Using Defocus Curves
}

\author{
Phillip J. Buckburst, ${ }^{1,2}$ James S. Wolfsobn, ${ }^{1}$ Shehzad A. Naroo, ${ }^{1}$ Leon N. Davies, ${ }^{1}$ \\ Gurpreet K. Bhogal, ${ }^{1}$ Athina Kipioti, ${ }^{3}$ and Sunil Shab ${ }^{3}$
}

Purpose. To determine the most appropriate analysis technique for the differentiation of multifocal intraocular lens (MIOL) designs by using defocus curve assessment of visual capability.

METHODs. Four groups of 15 subjects were implanted bilaterally with either monofocal intraocular lenses, refractive MIOLs, diffractive MIOLs, or a combination of refractive and diffractive MIOLs. Defocus curves between $-5.0 \mathrm{D}$ and $+1.5 \mathrm{D}$ were evaluated by using an absolute and relative depth-of-focus method, the direct comparison method, and a new "area-offocus" metric. The results were correlated with a subjective perception of near and intermediate vision.

REsults. Neither depth-of-focus method of analysis was sensitive enough to differentiate between MIOL groups $(P>$ 0.05). The direct comparison method indicated that the refractive MIOL group performed better at +1.00 diopter (D), $-1.00 \mathrm{D}$, and $-1.50 \mathrm{D}$ and worse at $-3.00 \mathrm{D},-3.50 \mathrm{D},-4.00 \mathrm{D}$, and $-5.00 \mathrm{D}$ than did the diffractive MIOL group $(P<0.05)$. The area-of-focus intermediate zone was greater with the refractive than with the diffractive MIOL group $(P=0.005)$ and the near zone was better with the diffractive $(P=0.020)$ and "mix and match" $(P=0.039)$ groups than with the refractive MIOL group. The subjective perception of intermediate and near vision agreed best with the area-of-focus metric for the intermediate $\left(r_{\mathrm{s}}=0.408, P=0.010\right)$ and near zone $\left(r_{\mathrm{s}}=0.484\right.$, $P<0.001)$

Conclusions. Conventional depth-of-focus metrics provide a single value to quantify the useful range of vision; however, they fail to provide sufficient detail to differentiate between MIOL designs. The direct comparison method provides a large amount of information, although the results can be complex to interpret. The proposed area-of-focus metric provides a simple, but differentiating method of evaluating MIOL defocus curves. (Invest Ophthalmol Vis Sci. 2012;53:3920-3926) DOI: 10.1167/iovs.11-9234

From the ${ }^{1}$ Ophthalmic Research Group, School of Life and Health Sciences, Aston University, Birmingham, United Kingdom; the ${ }^{2}$ School of Health Professions, Plymouth University, Plymouth, United Kingdom; and the ${ }^{3}$ Midland Eye Institute, Solihull, Birmingham, United Kingdom.

Submitted for publication December 3, 2011; revised April 15, 2012; accepted May 1, 2012.

Disclosure: P.J. Buckhurst, None; J.S. Wolffsohn, None; S.A. Naroo, None; L.N. Davies, None; G.K. Bhogal, None; A. Kipioti, None; S. Shah, Abbott Medical Optics, Inc. (F), Lenstec (F)

Corresponding author: Phillip J. Buckhurst, School of Health Professions, Peninsula Allied Health Centre, Derriford Road, Plymouth University, Plymouth, UK, PL6 8BH;

phillip.buckhurst@plymouth.ac.uk.
$\mathrm{M}$ ultifocal intraocular lenses (MIOLs) use the principle of simultaneous vision, whereby light is split into two or more focal points to extend the range of clear vision. MIOLs do not alter optically with attempted eye focus and hence objective in vivo methods for assessing accommodation, such as dynamic aberrometry ${ }^{1}$ and dynamic autorefraction, ${ }^{2,3}$ are inappropriate.

Previous in vivo MIOL studies have examined the visual range of MIOLs by using measurements of visual acuity (VA) either at varying distances ${ }^{4-6}$ or through different levels of spectacle lens defocus. ${ }^{7-9}$ However, the more physical method of measuring VA at varying distances is often impractical owing to the need to control angular image size and luminance.

Measuring VA through a range of spectacle lenses creates a defocus curve profile. A previous study has suggested that VA measured with this method provides an underestimation of the true VA at the corresponding distance owing to the magnification effects of lenses in the spectacle plane (although this can be compensated for mathematically ${ }^{10}$ ) and the disrupted natural associated convergence and pupil response. ${ }^{11}$

The two focal points created by the conventional bifocal MIOL result in a distinctive defocus curve profile with two peaks of optimum acuity: one at the distance focal point and the other at the near focus. ${ }^{6}$ Hence, defocus curves demonstrate the strength of the near addition (the separation in diopters between the distance and near peak) as well as the quality of vision at each dioptric level of spectacle defocus. ${ }^{12}$

There is variation both in the methods used to measure defocus curves ${ }^{13}$ and in the approaches taken to analyze the results. ${ }^{10}$ The direct comparison method of analysis involves statistical comparison of the visual acuity at each defocus level; the linked nature of repeated measurements needs to be accounted for statistically and the large number of comparisons can complicate clinical interpretation. Alternatively, the depth-of-focus method of analysis describes the dioptric range over which the subjects can sustain a specific absolute or relative level of VA. There is no consensus over the level of acuity considered to be appropriate for depth-of-focus measurements, and as this criterion is not always stated, the disparity of methodology prevents meaningful comparisons between studies. A relative criterion for depth-of-focus analysis defines the VA cutoff relative to the best-attained level of VA; relative criteria have not been used in multifocal studies but have been used with the assessment of accommodating IOLs. ${ }^{3}$ An absolute criterion identifies the limits of VA independent to the best-attained VA; the limit of 0.3 LogMAR is the most common criterion used with multifocal IOL studies and matches the level of VA defined as the driving standard in Europe $^{14}$ and in all but three states in the United States. ${ }^{15}$ The defocus curve of a MIOL can pass through the depth of focus criterion line several times; it is unclear how studies have resolved this previously as this possibility has not been addressed. 
TABLE 1. Subject Demographics for Each of the Bilaterally Implanted IOL Groups $(n=15$ in each)

\begin{tabular}{|c|c|c|c|c|}
\hline & Bilateral Monofocal IOL & Bilateral ReZoom & Bilateral Tecnis ZM900 & Mix and Match \\
\hline Age, $y$, Mean $\pm S D$ & $62.1 \pm 6.8$ & $62.3 \pm 8.4$ & $60.7 \pm 11.0$ & $58.5 \pm 9.2$ \\
\hline Sex & 3 male, 12 female & 7 male, 8 female & 4 male, 11 female & 7 male, 8 female \\
\hline
\end{tabular}

Given the lack of standardization in the methods used to analyze a defocus curve, the aim of this study was to determine suitable metrics for the stratification of MIOL defocus curves.

\section{MeTHODS}

\section{Subjects and Measurements} years, scheduled for bilateral cataract surgery or clear lens extraction, were recruited from Solihull Hospital and the Midland Eye Institute (Solihull, UK). The inclusion criteria were the absence of any ocular pathologic finding and previous surgery, corneal astigmatism $\leq 1.50 \mathrm{D}$, and willingness to participate within the study. The subjects were sequentially allocated to one of four groups in accordance with the date at which they attended for their preoperative assessment; as such, the subjects were randomly assigned by the date at which they first attended the surgical center: the first group of 15 subjects was bilaterally implanted by using a mix-and-match strategy; a ReZoom the right eye and a Tecnis ZM900 MIOL (Abbott Medical Optics) was implanted in the left. The second group of 15 subjects was implanted bilaterally with the ReZoom MIOL. The third group of 15 subjects was implanted bilaterally with the Tecnis ZM900 MIOL. The fourth group of 15 subjects was implanted bilaterally with a spherical monofocal IOL (Softec 1; Lenstec, St. Petersburg, FL) with power optimized for distance vision.

All patients gave informed consent to participate in the study, following explanation of the procedures and the risks involved. The study adhered to the tenets of the Declaration of Helsinki and was approved by the local research ethics committee. The subject demographics are detailed in Table 1.

Preoperatively, an IOLMaster (Carl Zeiss Meditec AG, Jena, Germany) running version 5.0 analysis software and NIDEK OPD-Scan II (Optical Path Difference Scanning System II; NIDEK Co., Ltd., Gamagori, Japan) aberrometer and topographer were used to determine axial length and corneal power. To determine IOL power, the Hoffer Q IOL formula was used for short axial lengths $(<22 \mathrm{~mm})$
Sixty subjects (21 males, 39 females) of mean age \pm SD $61.2 \pm 8.9$ MIOL (Abbott Medical Optics, Inc., Santa Ana, CA) was implanted in

and the SRK/T was used for all other axial lengths; emmetropia was the target in all cases.

All operations were performed by one of two surgeons using topical or local anesthetic. A 2.85 -mm clear corneal incision (widening to $3.2 \mathrm{~mm}$ before the insertion of the IOL) was placed on the steepest corneal axis to reduce residual levels of postoperative astigmatism. Phacoemulsification, aspiration, and irrigation were performed through a 5.5-mm capsulorhexis with the Millennium phacoemulsification system (Bausch and Lomb, Rochester, NY). All IOLs were implanted into the capsular bag.

The ReZoom is a hydrophobic acrylic MIOL, with a 6-mm five-zone concentric refractive optic. The IOL has an equivalent near addition of $+3.50 \mathrm{D}$ at the IOL plane (approximately $+2.60 \mathrm{D}$ at the spectacle plane) and an aberration control aspheric posterior surface optic. The Tecnis ZM900 is a hydrophobic acrylic MIOL. The 6-mm optic has a concentric fully diffractive anterior surface and an aberration control posterior optic. The diffractive first-order power is equivalent to a near addition of $+4.00 \mathrm{D}$ at the IOL plane (approximately $+3.20 \mathrm{D}$ at the spectacle plane). The monofocal Softec $1 \mathrm{IOL}$ involved in the study is a one-piece hydrophilic acrylic spherical IOL with a $5.75-\mathrm{mm}$ optic.

All subjects were examined 3 to 6 months postoperatively for the purposes of this study. No clinically significant posterior capsule opacity was present at the time of measurement. Subjective manifest refraction, maximizing the positive power while maintaining optimum distance VA, was performed at $6 \mathrm{~m}$ by a single UK-qualified optometrist (P.J.B.). Monocular and binocular uncorrected and best-corrected distance VA was measured by using a computerized test chart (Test Chart 2000; Thomson Software Solutions, Hatfield, UK), and monocular and binocular intermediate $(79 \mathrm{~cm})$ and near $(40 \mathrm{~cm})$ VA was measured with the Early Treatment of Diabetic Retinopathy Study (ETDRS) Near LogMAR Chart 2000 (Precision Vision, LaSalle, IL). As several measurements of near and intermediate vision were required for the study, four EDTRS charts were cycled to prevent memorization of letters.

Binocular distance corrected defocus curves were measured in each subject with the Test Chart 2000, positioned at $6 \mathrm{~m}$, to measure the VA with each defocus lens. These were sequenced in a random order over the range of +1.50 to $-5.00 \mathrm{D}$ in $0.50-\mathrm{D}$ steps with the letters on the Test Chart 2000 randomized between measures. An Oculus Universal Trial Frame (Keeler Ltd., Windsor, UK), adjusted to

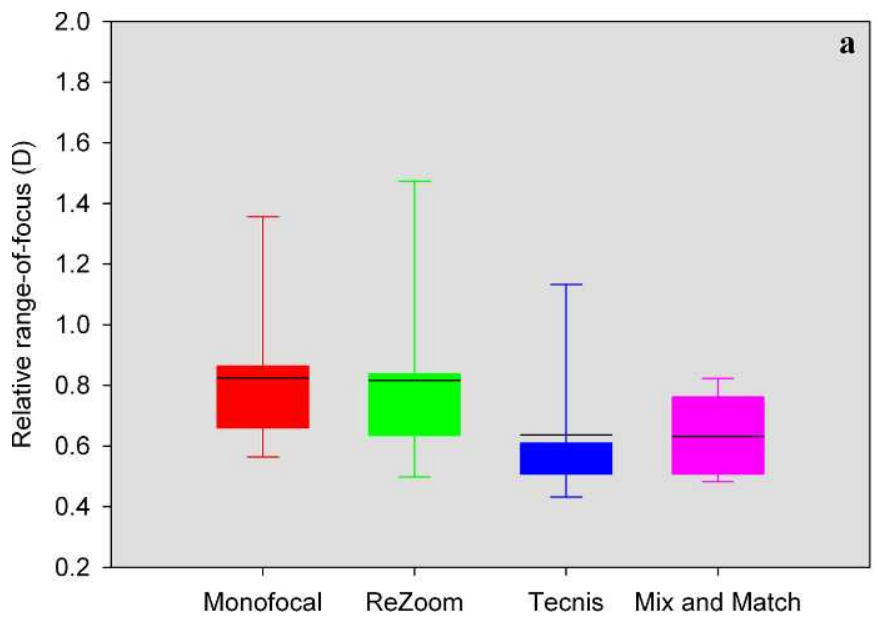

Figure 1. Results of the relative (a) and absolute (b) depth-of-focus methods of analysis 
TABLE 2. Coefficient of Determination $\left(r^{2}\right)$ and Standard Error of Estimate in Fitting the Defocus Curve Data with Increasing Order of Polynomial $(n=60)$

\begin{tabular}{lcc}
\hline & $\boldsymbol{r}^{2}$ & $\begin{array}{c}\text { Standard Error } \\
\text { of Estimate }\end{array}$ \\
\hline 4th order & $0.8197 \pm 0.1404$ & $0.0964 \pm 0.0410$ \\
5th order & $0.8458 \pm 0.1317$ & $0.0928 \pm 0.0410$ \\
6th order & $0.9250 \pm 0.0652$ & $0.0705 \pm 0.0301$ \\
7th order & $0.9507 \pm 0.0444$ & $0.0601 \pm 0.0253$ \\
8th order & $0.9635 \pm 0.0371$ & $0.0555 \pm 0.0222$ \\
9th order & $0.9768 \pm 0.0243$ & $0.0493 \pm 0.0208$ \\
10th order & $0.9315 \pm 0.2166$ & $0.1548 \pm 0.4906$ \\
\hline
\end{tabular}

ensure a 12-mm back vertex distance, was used to house the manifest refraction and each additional defocus lens. For each measurement of VA, subjects were prompted once with the phrase "can you read any more letters on the line below"? according to the methodology described by Gupta and colleagues. ${ }^{10}$ Each subject also subjectively rated his or her intermediate and near vision on a scale of 0 (completely unsatisfied) to 5 (completely satisfied). Monocular pupil sizes were measured by using a validated portable infrared pupillography device, the Pupilscan II infrared pupillometer (Keeler Ltd.). ${ }^{16,17}$

\section{Statistical Analysis}

The one-sample Kolmogorov-Smirnov test was used to determine if results from each measurement followed a normal distribution. Where the data followed a normal distribution, parametric analysis was performed, whilst nonparametric statistical analysis was used for nonnormally distributed data. ${ }^{18}$ All defocus curve acuities were corrected for spectacle magnification (SM) according to a back vertex distance (BVD) of $12.0 \mathrm{~mm}$ (equation $1^{10}$ ).

$$
\mathrm{SM}=\text { Lens Power } /[1(\mathrm{BVD} \times \text { Lens Power })]
$$

For each defocus curve, a best-fit polynomial regression curve was fitted to the data points with SigmaPlot 2000 (SPSS Inc., Chicago, IL) (y is the visual acuity [LogMAR] and $\mathrm{x}$ is the optical defocus [diopters]). Each data set (14 points) was fitted with a 4th, 5th, 6th, 7th, 8th, 9th, 10th, 11th, and 12th order polynomial. The curve-fitting process was limited to 200 iterations for each curve. Increasing the order of the polynomial to fit the defocus curves resulted in a higher $r^{2}$ and decreased the standard error of estimate up until a ninth order polynomial; fitting polynomials of 10 th order displayed more variability in the standard error of estimation, as valid curves could not be fitted to all data sets within the iteration limit (Table 2). Therefore, a ninth order polynomial function was used for all further analysis as it was the lowest-order polynomial that provided a universal best fit to all data sets (equation 2).

$$
y=a+b x+c x^{2}+d x^{3}+e x^{4}+f x^{5}+g x^{6}+b x^{7}+i x^{8}+j x^{9}
$$

$\mathrm{VA}$ at $40 \mathrm{~cm}$, measured with the near EDTRS chart, was compared with the VA with a defocus lens of $-2.50 \mathrm{D}$, using Pearson's product moment correlation and Bland and Altman limits of agreement. ${ }^{19}$

A relative range of focus was calculated for each subject by determining when the polynomial dropped below +0.04 LogMAR greater than the patient's best VA as described by Gupta and
TABLE 4. Best Distance-Corrected Intermediate $(79 \mathrm{~cm})$ and Near $(40$ cm) LogMAR VA for Each of the Bilaterally Implanted IOL Groups $(n=$ 15 in each)

\begin{tabular}{ccccc}
\hline Binocular & Binocular & \\
Softec 1 & $\begin{array}{c}\text { Binocular } \\
\text { ReZoom }\end{array}$ & $\begin{array}{c}\text { Tecnis } \\
\text { ZM900 }\end{array}$ & $\begin{array}{c}\text { Mix and } \\
\text { Match }\end{array}$ \\
\hline $40 \mathrm{~cm}$ & $+0.57 \pm 0.09$ & $+0.26 \pm 0.10$ & $+0.17 \pm 0.11$ & $+0.18 \pm 0.10$ \\
$79 \mathrm{~cm}$ & $+0.28 \pm 0.09$ & $+0.17 \pm 0.10$ & $+0.28 \pm 0.14$ & $+0.19 \pm 0.08$ \\
\hline
\end{tabular}

colleagues. ${ }^{10}$ If the second multifocal peak (corresponding with the near focal point) also met these criteria, the range of defocus values meeting the criteria for both focal points was summated. The absolute criteria of 0.30 LogMAR was also used to calculate depth of focus; the Newton-Raphson method ${ }^{20}$ was used to calculate $x$ when $y=0.3$. The Newton-Raphson method is used to find the roots of a function, by adjusting the polynomial function by 0.3 to find $x$ when $y=0.3$ (equation 3). The table of corresponding $x$ and $y$ values produced by Sigmaplot 2000 was used to determine the initial approximation $x_{0}$.

$$
\begin{aligned}
x_{1}= & x_{0}=-\frac{f\left(x_{0}\right)}{f^{\prime}\left(x_{0}\right)} \\
= & x_{0} \\
& -\frac{(a-0.3)+b x_{0}+c x_{0}^{2}+d x_{0}^{3}+e x_{0}^{4}+F x_{0}^{5}+g x_{0}^{6}+b x_{0}^{7}+i x_{0}^{8}+j x_{0}^{9}}{b+2 c x_{0}+3 d x_{0}^{2}+4 e x_{0}^{3}+5 f x_{0}^{4}+6 g x_{0}^{5}+7 b x_{0}^{6}+8 i x_{0}^{7}+9 j x_{0}^{8}}
\end{aligned}
$$

The resultant $x_{1}$ from equation 3 is a better approximation of $x$ when $\mathrm{y}$ $=0.3$; however, for increased accuracy this process is repeated by taking the resultant $x_{1}$ to be $x_{n}$ and putting this value through equation 4 until the percentage error (\% error) is reduced to 0 (equation 5)

$$
\begin{aligned}
x_{n+1}= & x_{n}=-\frac{f\left(x_{n}\right)}{f^{\prime}\left(x_{n}\right)} \\
= & x_{n} \\
& -\frac{(a-0.3)+b x_{n}+c x_{n}^{2}+d x_{n}^{3}+e x_{n}^{4}+F x_{n}^{5}+g x_{n}^{6}+b x_{n}^{7}+i x_{n}^{8}+j x_{n}^{9}}{b+2 c x_{n}+3 d x_{n}^{2}+4 e x_{n}^{3}+5 f x_{n}^{4}+6 g x_{n}^{5}+7 b x_{n}^{6}+8 i x_{n}^{7}+9 j x_{n}^{8}} \\
& \% \text { error }=\frac{\left(x_{n+1}-x_{n}\right)}{\left(\frac{x_{n+1}+x_{n}}{2}\right)}
\end{aligned}
$$

The Newton-Raphson method was used to determine each intersection of the curve at 0.3 LogMAR. The range of focus was calculated as the dioptric distance over which VA was better than 0.3 LogMAR.

The polynomial equations for each curve were integrated so that a new "area of focus" metric $\left(\operatorname{LogMAR} * \mathrm{~m}^{-1}\right)$ could be derived (equation

\begin{tabular}{|c|c|c|c|c|c|c|c|c|}
\hline & \multicolumn{2}{|c|}{ Softec 1} & \multicolumn{2}{|c|}{ ReZoom } & \multicolumn{2}{|c|}{ Tecnis ZM900 } & \multicolumn{2}{|c|}{ Mix and Match } \\
\hline & $\mathbf{R}$ & $\mathbf{L}$ & $\mathbf{R}$ & $\mathbf{L}$ & $\mathbf{R}$ & $\mathbf{L}$ & Tecnis & ReZoom \\
\hline $\mathrm{mm}$, mean $\pm \mathrm{SD}$ & $4.23 \pm 0.74$ & $4.27 \pm 0.78$ & $4.09 \pm 0.71$ & $4.06 \pm 0.69$ & $4.16 \pm 0.62$ & $4.16 \pm 0.61$ & $4.18 \pm 0.76$ & $4.19 \pm 0.77$ \\
\hline
\end{tabular}
6). In accordance with the consensus of previous literature, the upper limit for depth of focus was defined as 0.3 LogMAR, corresponding with the European ${ }^{14}$ and American ${ }^{15}$ binocular visual acuity driving standards.

$$
\int_{a_{1}}^{a_{0}} a x+b x^{2}+c x^{3}+d x^{4}+e x^{5}+f x^{6}+g x^{7}+b x^{8}+i x^{9}+b x^{10}
$$

The defocus curves were divided into distance, intermediate, and near

TABLE 3. Pupil Sizes for Each of the Bilaterally Implanted IOL Groups ( $n=15$ in each)

L, left; R, right. 

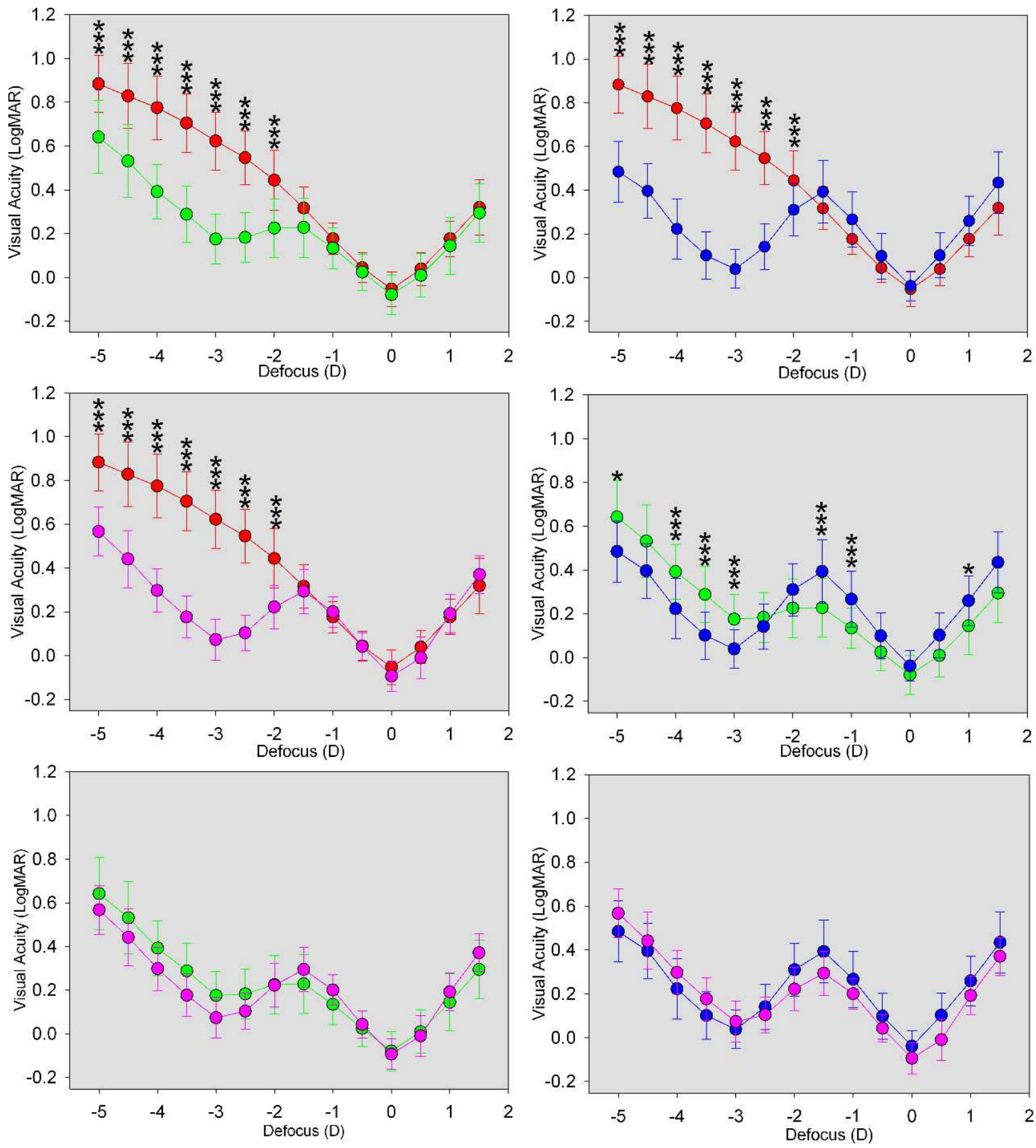

- Monofocal O- ReZoom

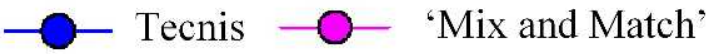

Figure 2. Results of the direct comparison method of analysis. ${ }^{* * *}$ Significance at the $P<0.01$ level; *significance at $P<0.05$.

zones. The near zone was defined as between -4.00 and $-2.00 \mathrm{D}$, corresponding with a 25 - to $50-\mathrm{cm}$ range, commonly referred to as the range of near vision. ${ }^{21}$ The intermediate zone was defined as -2.00 to $-0.50 \mathrm{D}$, from $50 \mathrm{~cm}$ (approximately arm's length) to $2 \mathrm{~m}$. Beyond this, the distance zone was defined as the distances between -0.50 to +0.50 D. These zones were used to define the limits of integration. A two-way repeated measures ANOVA was used to determine if there was any statistically significant difference in the area-of-focus and the defocus curves between lenses. If a significant difference was found, then a one-way ANOVA was used to examine differences by applying Bonnferoni post hoc tests to determine pairwise differences.

The subjective rating of intermediate vision was correlated with the absolute, relative, and area (intermediate) defocus curve metrics, as well as with best distance-corrected intermediate VA $(79 \mathrm{~cm})$, by using the Spearman's rank correlation coefficient. The subjective rating of near vision was correlated with the absolute, relative, and area (near) defocus curve metrics, as well as with best distance-corrected near VA (at $40 \mathrm{~cm}$ ), by using the Spearman's rank correlation coefficient. 


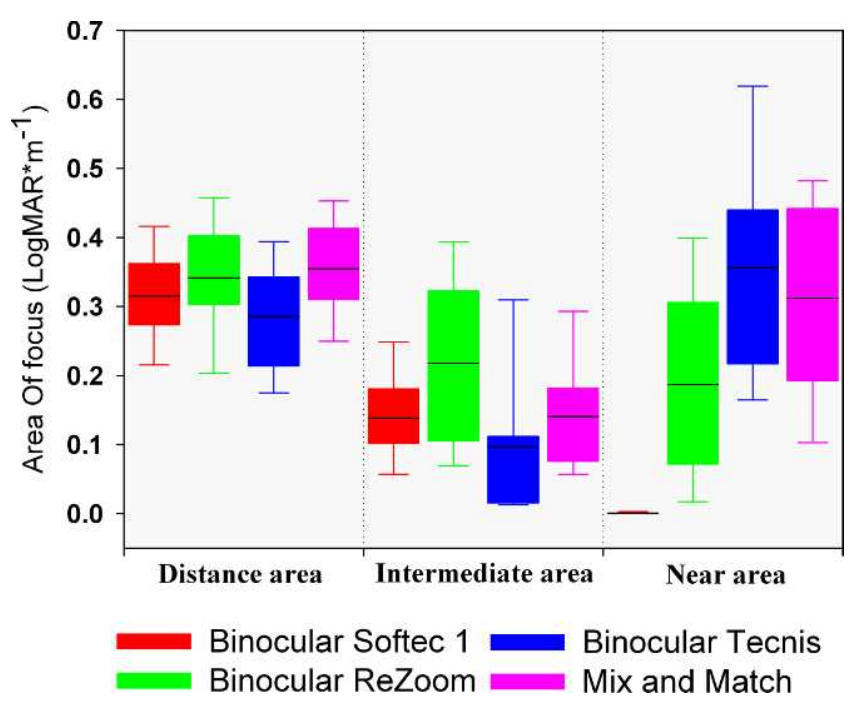

Figure 3. Results of the area-of-focus method of analysis for the distance, intermediate, and near zone.

\section{Results}

The IOL-implanted groups had similar demographics (age: $F_{3}=$ $0.370, P=0.829$; Table 1).

No significant difference in the pupil size of each group was found for both the right eye $\left(F_{3}=0.094, P=0.963\right)$ and left eye $\left(F_{3}=0.227, P=0.878\right.$ ) (Table 3).

Measurement of best distance-corrected near VA (at $40 \mathrm{~cm}$ ) revealed greater VA for all multifocal groups than for the monofocal group $(P<0.001)$; however, no differences were found between the MIOL groups $(P>0.05)$. For best distancecorrected intermediate VA $(79 \mathrm{~cm})$, the ReZoom MIOL group achieved higher levels of intermediate VA than both the monofocal $(P=0.019)$ and Tecnis ZM900 $(P=0.024)$ groups and similar levels of intermediate VA in comparison with the mix-and-match group $(P=0.419)$ (Table 4$)$.

The relative depth-of-focus analysis method provided similar results for all lens types $\left(F_{3}=2.144, P=0.105\right.$; Fig. 1$)$.

The results obtained with the absolute depth-of-focus analysis method revealed that all multifocal groups demonstrated a greater depth of focus than a monofocal group $(P<$ 0.001 ). However, this method of analysis found no significant differences in the results for each of the MIOL groups $(P>$ 0.05 ; Fig. 1).

The results of the direct comparison method revealed that all of the MIOL groups achieved significantly higher VAs over the range of -2.00 to $-5.00 \mathrm{D}$ than did a monofocal IOL group $(P<0.001)$. In addition, this method revealed differences between the MIOL designs, showing that the binocular ReZoom performed better at $+1.00 \mathrm{D}(P=0.024),-1.00 \mathrm{D}$ $(P=0.002)$, and $-1.50 \mathrm{D}(P=0.003)$ than the binocular Tecnis ZM900 group but performed significantly worse at $-3.00 \mathrm{D}(P$ $=0.006),-3.50 \mathrm{D}(P<0.001),-4.00 \mathrm{D}(P=0.003)$, and -5.00 $\mathrm{D}(P=0.017)$. The mix-and-match group showed similar results to both the ReZoom and Tecnis groups (Fig. 2).

The area-of-focus analysis method revealed similar results between all groups for the distance area $\left(F_{3}=2.541, P=\right.$ $0.065)$. For the intermediate zone, the ReZoom group had better vision (a larger area) than the Tecnis ZM900 group $(P=$ $0.005)$; no other differences were found for the intermediate zone. For the near area of focus, all MIOL groups achieved better vision than the monofocal IOL group $(P<0.001)$; in

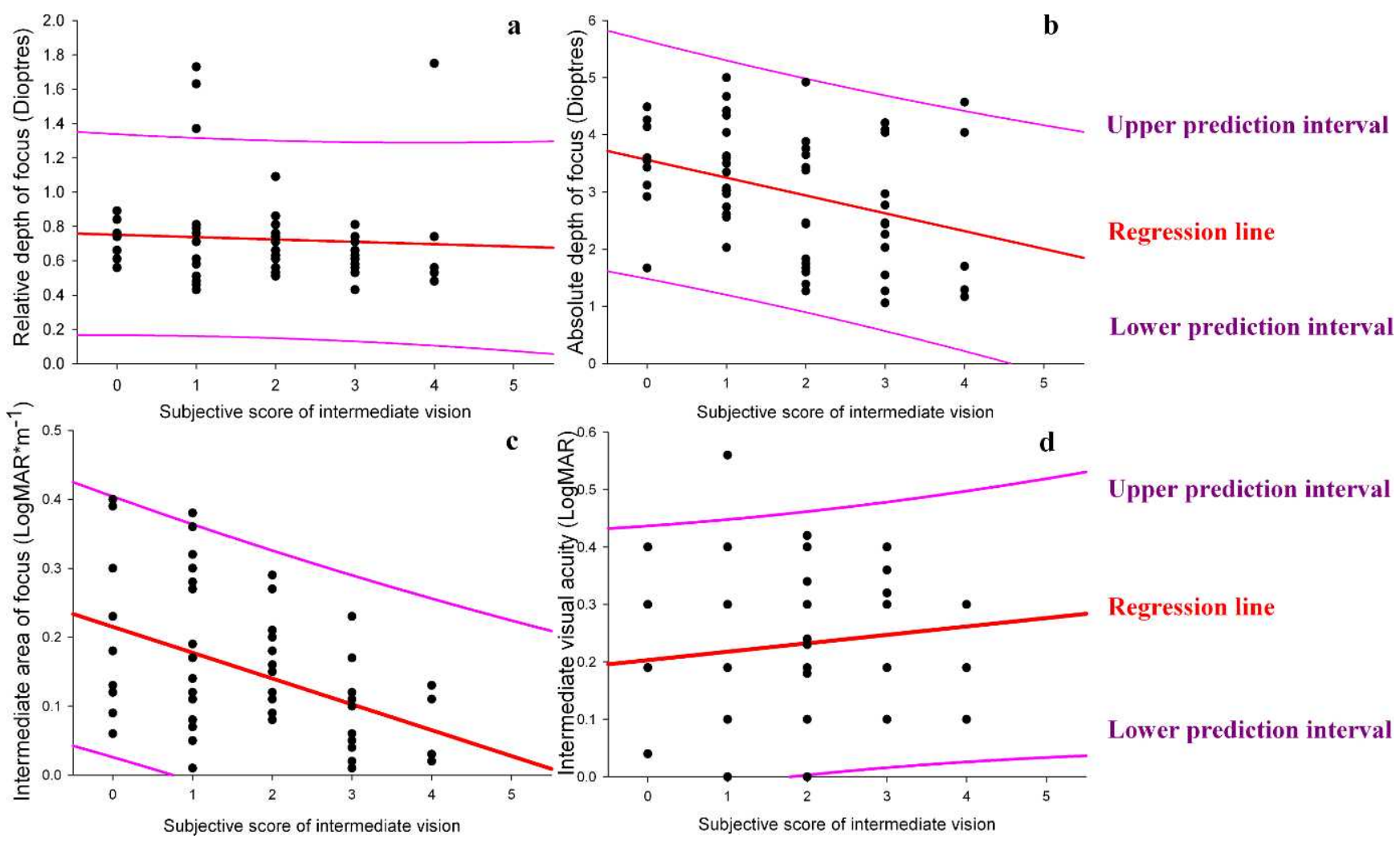

FiguRE 4. Correlation of the subjective perception of intermediate vision with (a) relative depth of focus, (b) absolute depth of focus, (c) intermediate area of focus, and (d) intermediate VA. 
addition the near area of the Tecnis ZM900 $(P=0.020)$ and of the mix-and-match $(P=0.039)$ groups was greater than that that of the ReZoom group (Fig. 3).

The intermediate area of focus demonstrated a higher correlation with the subjective rating of intermediate vision $\left(r_{\mathrm{s}}\right.$ $=0.408, P=0.010)$ than did the best distance-corrected intermediate VA $\left(r_{\mathrm{s}}=0.148, P=0.204\right)$, relative range of focus $\left(r_{\mathrm{s}}=0.36, P=0.783\right)$, and absolute range of focus $\left(r_{\mathrm{s}}=0.340, P\right.$ $=0.008$ ) (Fig. 4). Similarly, the subjective rating of near vision correlated strongest with the near area of focus $\left(r_{\mathrm{s}}=0.484, P<\right.$ $0.001)$ in comparison with best distance-corrected near VA $\left(r_{\mathrm{s}}\right.$ $=0.385, P=0.001)$, relative range of focus $\left(r_{\mathrm{s}}=0.154, P=\right.$ $0.241)$, and absolute range of focus $\left(r_{\mathrm{s}}=0.408, P=0.001\right)$ (Fig. 5).

\section{Discussion}

Direct comparisons of robustly measured defocus curves provide a detailed comparison of VA at every level of defocus. ${ }^{10,13}$ However, metrics for providing a global overview of the performance of a lens are important to allow standardized comparisons between studies. Relative and absolute depth-of-focus analysis methods are the two most common metrics used to assess defocus curves. However, in this study, neither the relative nor the absolute defocus curve analysis method was sensitive to differences between MIOL designs despite the clear variation that was demonstrated with the direct comparison method and area-of-focus method.

The area-of-focus metric provides an overview of the visual range separately for distance, near, and intermediate. By using area it also accounts for the level of VA within the range as well as the range itself. The area metric identified the increased level of VA within the intermediate range of a lower addition
MIOL in comparison to a high addition lens. With the area metric, differences between the MIOL groups can be identified as well as the difference between a monofocal IOL and MIOL. The area metric provides an overview of results that can be standardized and are sensitive to differences between MIOL designs

A potential source of error occurs when fitting polynomial curves to each data set. This study concluded that when using a 14-point data set between -5.0 and $+1.5 \mathrm{D}$, a ninth order polynomial is most appropriate. Fitting polynomials of a high order creates oscillation of the fitted curve, known as the Runge phenomenon. ${ }^{22}$ This oscillation occurs mostly at the edges of a data set between the first and last values. To account for this phenomenon, the chosen defocus curve range was $1.00 \mathrm{D}$ on either side of the required range for measurement of the area of focus. Therefore, when using a ninth order polynomial, it is important to retain the full range of defocus curve between -5.0 and $+1.5 \mathrm{D}$ despite the area metric using only the area between -4.0 and $+0.5 \mathrm{D}$.

Near VA is the most common method used to determine the effectiveness of a MIOL. The intermediate and near area of focus demonstrated a higher correlation with the subjective rating of near vision than did intermediate and near VA, possibly owing to the wide focal range with which the area metric is generated, better capturing the subjective experience.

In conclusion, defocus curve methodology and analysis need to be standardized so that results can be compared between studies. The direct method of assessment is important as it can determine differences between lenses at each level of focus. However, the results attained from this method need to be viewed with caution as the number of statistical tests required to analyze a defocus curve leaves it vulnerable to type 1 errors. Alternatively, this study proposed an area-of-focus
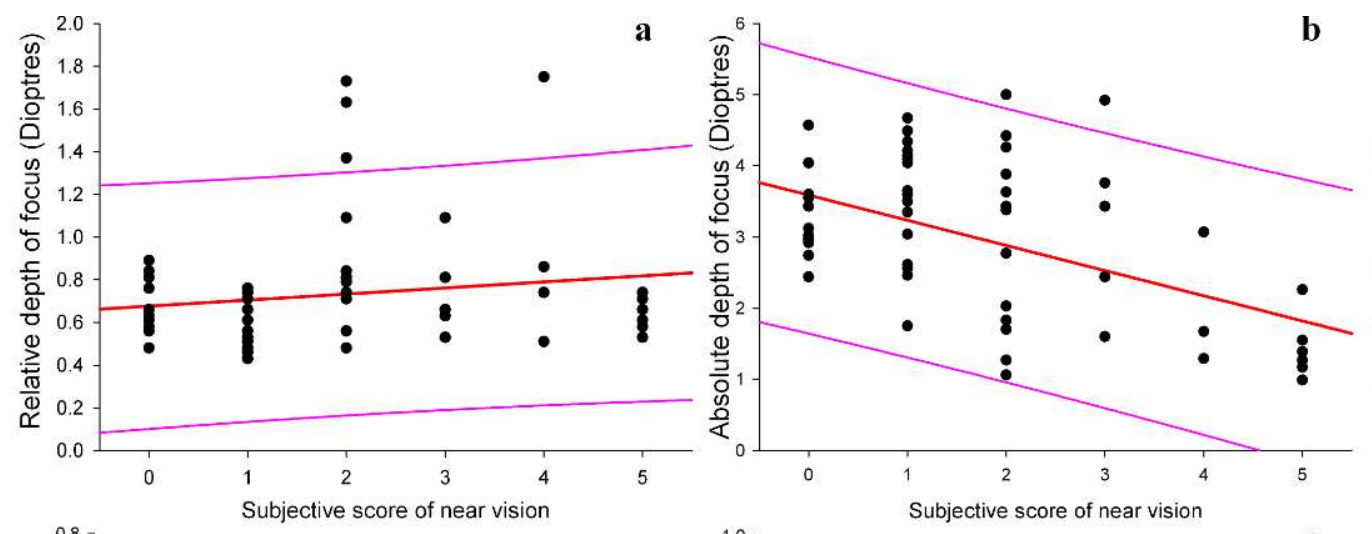

Upper prediction interval
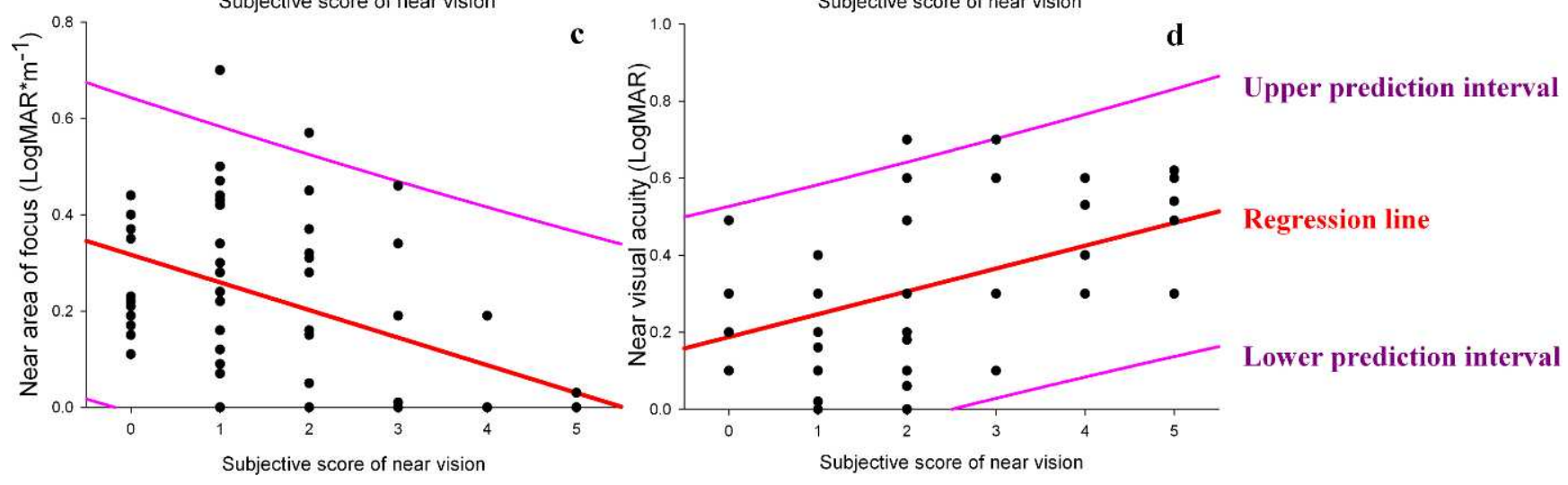

Figure 5. Correlation of the subjective perception of near vision with (a) relative depth of focus, (b) absolute depth of focus, (c) near area of focus, and (d) near VA. 
metric that has been shown to differentiate between multifocal IOL designs and to correlate most strongly with subjective experience.

\section{References}

1. Wolffsohn JS, Davies LN, Gupta N, et al. Mechanism of action of the Tetraflex accommodative intraocular lens. $J$ Refract Surg. 2010;26:858-862.

2. Wolffsohn JS, Naroo SA, Motwani NK, et al. Subjective and objective performance of the Lenstec KH-3500 "accommodative" intraocular lens. Br J Ophthalmol. 2006;90:693-696.

3. Cleary G, Spalton DJ, Marshall J. Pilot study of new focus-shift accommodating intraocular lens. J Cataract Refract Surg. 2010;36:762-770.

4. Schmidinger G, Geitzenauer W, Hahsle B, Klemen U, Skorpik C, Pieh S. Depth of focus in eyes with diffractive bifocal and refractive multifocal intraocular lenses. J Cataract Refract Surg. 2006;32:1650-1656.

5. Hayashi K, Manabe S, Hayashi H. Visual acuity from far to near and contrast sensitivity in eyes with a diffractive multifocal intraocular lens with a low addition power. J Cataract Refract Surg. 2009;35:2070-2076.

6. Maxwell WA, Cionni RJ, Lehmann RP, Modi Ss. Functional outcomes after bilateral implantation of apodized diffractive aspheric acrylic intraocular lenses with $\mathrm{a}+3.0$ or +4.0 diopter addition power: randomized multicenter clinical study. $J$ Cataract Refract Surg. 2009;35:2054-2061.

7. Steinert RF, Post CF, Brint SF, et al. A prospective, randomized, double-masked comparison of a zonal-progressive multifocal intraocular lens and a monofocal intraocular lens. Ophthalmology. 1992;99:853-860.

8. Cillino S, Casuccio A, Di Pace F, et al. One-year outcomes with new-generation multifocal intraocular lenses. Ophthalmology. 2008;115:1508-1516.

9. Alfonso JF, Fernández-Vega L, Amhaz H, Montés-Micó R, Valcárcel B, Ferrer-Blasco T. Visual function after implantation of an aspheric bifocal intraocular lens. J Cataract Refract Surg. 2009;35:885-892.
10. Gupta N, Wolffsohn JS, Naroo SA. Optimizing measurement of subjective amplitude of accommodation with defocus curves. J Cataract Refract Surg. 2008;34:1329-1338.

11. Pieh S, Kellner C, Hanselmayer G, et al. Comparison of visual acuities at different distances and defocus curves. J Cataract Refract Surg. 2002;28:1964-1967.

12. Hansen TE, Corydon L, Krag S, Thim K. New multifocal intraocular lens design. J Cataract Refract Surg. 1990;16:3841.

13. Gupta N, Naroo SA, Wolffsohn JS. Is randomisation necessary for measuring defocus curves in pre-presbyopes? Cont Lens Anterior Eye. 2007;30:119-124.

14. Coeckelbergh TRM, Brouwer WH, Cornelissen FW, Wolffelaar PV, Kooijman AC. The effect of visual field defects on driving performance: a driving simulator study. Arch Ophthalmol. 2002;120:1509-1516.

15. Colenbrander A, De Laey J-J; International Council of Ophthalmology. Visual standards-vision requirements for driving safety. Prepared for the 30th World Ophthalmology Congress; 2006:1-25; Sao Paulo, Brazil.

16. Murray A, Lawrence GP, Clayton RH. Repeatability of dynamic eye pupil response measurement using the Pupilscan instrument. Clin Phys Physiol Meas. 1991;12:377-385.

17. Scheffel M, Kuehne C, Kohnen T. Comparison of monocular and binocular infrared pupillometers under mesopic lighting conditions. J Cataract Refract Surg. 2010;36:625-630.

18. Armstrong RA, Davies LN, Dunne MCM, Gilmartin B. Statistical guidelines for clinical studies of human vision. Ophthalmic Physiol Opt. 2011;31:123-136.

19. Bland JM, Altman DG. Statistical methods for assessing agreement between two methods of clinical measurement. Lancet. 1986;1:307-310.

20. Ypma TJ. Historical development of the Newton-Raphson method. Soc Ind Appl Math. 1995;37:531-551.

21. Millodot M. Dictionary of Optometry and Visual Science. 7 th ed. London, UK: Butterworth Heinemann Inc.; 2002:401-402.

22. Runge $C$. Über empirische Funktionen und die Interpolation zwischen äquidistanten Ordinaten. Zeitschrift für Matbematik und Physik. 1901;46:224-243. Available at: www.archive. org. Accessed November 25, 2011. 